\title{
THE EFFECT OF TRACHEAL DIVERTICULUM AND CHRONIC OSTRUCTIVE LUNG DISEASE ON CHEST ANTHROPOMETRY
}

\author{
Mine ARGALI DENIZ ${ }^{1}$, Hilal ER ULUBABA ${ }^{2}$, M. Furkan ARPACI ${ }^{3}$, Fatih CAVUS ${ }^{3}$, \\ Gokhan DEMIRTAS ${ }^{4}$, Turgay KARATAS ${ }^{3}$, and Davut OZBAG ${ }^{3}$ \\ ${ }^{1}$ Suleyman Demirel University Research and Application Hospital \\ ${ }^{2}$ Yesilyurt Hasan Calık Hospital, Malatya, Turkey \\ ${ }^{3}$ Inonu University \\ ${ }^{4}$ Malatya Education and Research Hospital
}

February 5, 2021

\begin{abstract}
Objective: In this study, the effect of tracheal diverticula (TD) on chest anthropometry and its relation with chronic obstructive pulmonary disease (COPD) was evaluated. Method: Between January 2019 and March 2020, 995 patients who underwent chest CT were retrospectively analyzed and TD was detected in 31 cases. Group 1 is only TD, Group 2 is TD + COPD, Group 3 is only COPD, Group 4 is defined as control group. We measured the localization, size, the distance to carina and vocal cord of TDs. In all groups chest diameters at T4 and T9 levels were measured as transverse and vertical plans. Results: TDs detected mostly at the T2 and T3 levels. In Group 1 and Group 2, there was a statistically significant difference the distance to TD of vocal chords. A statistically significant difference was found between Group 1 and Group 3 only in the vertical diameter at the T4 and T9 levels. Conclusion: We observed that COPD effect TD location and also TD had opposite effect on anteroposteriorly increasing chest parameters in COPD. Precence of TD is essential on COPD patients about thorax anthropometry. Keywords: Tracheal diverticulum; antropometry; radiology; COPD; chest diameter
\end{abstract}

THE EFFECT OF TRACHEAL DIVERTICULUM AND CHRONIC OSTRUCTIVE LUNG DISEASE ON CHEST ANTHROPOMETRY

\section{SUMMARY}

Objective: In this study, the effect of tracheal diverticula (TD) on chest anthropometry and its relation with chronic obstructive pulmonary disease (COPD) was evaluated.

Method: Between January 2019 and March 2020, 995 patients who underwent chest CT were retrospectively analyzed and TD was detected in 31 cases. Group 1 is only TD, Group 2 is TD + COPD, Group 3 is only COPD, Group 4 is defined as control group. We measured the localization, size, the distance to carina and vocal cord of TDs. In all groups chest diameters at T4 and T9 levels were measured as transverse and vertical plans.

Results: TDs detected mostly at the T2 and T3 levels. In Group 1 and Group 2, there was a statistically significant difference the distance to TD of vocal chords. A statistically significant difference was found between Group 1 and Group 3 only in the vertical diameter at the T4 and T9 levels.

Conclusion: We observed that COPD effect TD location and also TD had opposite effect on anteroposteriorly increasing chest parameters in COPD. Precence of TD is essential on COPD patients about thorax 
anthropometry.

Keywords: Tracheal diverticulum; antropometry; radiology; COPD; chest diameter

Tracheal diverticulum (TD) is a benign condition of the tracheal wall that is rarely diagnosed clinically, and there are very few case reports of this condition in the literature. The longest axes of TDs can extend up to $3 \mathrm{~cm}$ and they can be single or multiple as oval peduncular cysts or sessile formations that can communicate with the trachea through channels (1-9). It has been reported that TD may occur as a result of congenital anomalies of the tracheobronchial tree or cavitation in a weak tracheal wall, and as a result of these two conditions, TD mainly differs in relation to the implantation site and the histological features of the wall (1- $6,8,10,11)$. Congenital TD is usually smaller than acquired TD and is more closely related to the trachea $(1-3,5,8,9,11-13)$. Congenital ones usually occur on the right side, $4-5 \mathrm{~cm}$ below the vocal cords or a few centimeters above the carina. Histologically, the respiratory epithelium resembles the tracheal wall containing smooth muscle and cartilage whereas acquired TD consists only of respiratory epithelium $(3,4,11$, $13,15)$. The cause of cartilage deficiency in the acquired form is thought to be due to increased intraluminal pressure or weakening of the structures after surgical procedures that destroy the mucous membrane from sensitive points in the trachea $(2,3,9-13)$. Thus, acquired TD can be seen at any level, although they typically occur along the right posterolateral wall near the thoracic inlet $(1,2,6,8,16,17)$.

TD is usually asymptomatic and therefore their frequency can be underestimated $(5,7,18)$. In most cases, diagnosis is made on the basis of features observed on X-rays, CT scans, bronchoscopy, and autopsies (1-3, $5-8,11-13,16,17,19)$. Consequently, the reported incidence varies depending on the diagnostic tool used. For example, in a postmortem study, the incidence was estimated to be about $1 \%$, compared with $0,3 \%$ in children diagnosed with bronchoscopy $(1,4,6-9,11,13,14)$. In a study using cervical CT, Buterbaugh \& Erly estimated that paratracheal air cyst (ie TD) occurred in approximately 3,7\% of the population (20).

Although it is rare and not specific, symptoms may develop in people with tracheal diverticula (3). Since the diverticulum usually acts as a reservoir for respiratory secretions, it may sometimes be associated with chronic cough and infection $(2-5,8,9,11,14)$. Although less common in patients with tracheal diverticulum, dyspnea, dysphagia, dysphonia, recurrent nerve palsy, cervical neck swelling, hematemesis, and hemoptysis have been described $(2,8,12)$. TD may also be associated with abnormal lung function and emphysema known as obstructive pulmonary disease (7-9). However, no relationship has been identified between TD and alpha-1-antitrypsin deficiency to date $(4,20)$.

$\mathrm{CT}$ has a great role in diagnosing TD. Because it gives information about the location, origin and size of the lesion, thus helping to distinguish congenital and acquired forms. Although fiberoptic bronchoscopy is beneficial, it can give false and negative results because it often misses the point of contact with the trachea $(3,4,6,10,11,14,17)$. Various approaches to the treatment of TD have been described, including surgical resection, endoscopic cauterization, and conservative measures. Most TDs are treated conservatively. Surgery is usually an option for truly symptomatic cases with larger diverticula or recurrent infections (1, $3,13)$. Conservative treatment with antibiotics, mucolytics and physiotherapy may be appropriate for asymptomatic, elderly or debilitated patients $(2,3,11,14)$.

\section{MATERIAL - METHOD}

This study was planned in accordance with the Declaration of Helsinki and approved by the Malatya Clinical Research Ethics Committee (Protocol code 2020-1260). 995 patients between the ages of 40-80 who underwent thorax computed tomography (CT) between January 2020 and March 2020 were retrospectively screened by a radiologist and 31 TDs were detected. CT scans were examinations taken on a spiral CT scanner and $1 \mathrm{~mm}$ slice thickness. The window width was 1200 Hounsfield Units (HU) for the parenchymal window and $350 \mathrm{HU}$ for the mediastinal window. Axial, coronal and sagittal plans were used for measurements.

Patients whose identity and demographic information were missing on CT images and whose investigated parameters were not evident were excluded from the study. Patients between the ages of 40-80 years without 
mediastinal injury or operation were included in the study. The localization, size, vocal cord and carina distance and level of all detected TDs were noted (Figure 1). In addition, the widest transverse chest diameter at the T4 vertebra and T9 vertebra levels and the widest anteroposterior chest diameters in both hemithoraces were measured and the mean was calculated (Figure 2).

These patients were divided into two separate groups as Group 1 only TD and Group 2 TD + COPD. Group 3 , only COPD, Group 4 (control group), was formed from healthy individuals of the same age and gender with thoracic CTs of the same dates. The relationship of chest diameters with TD and COPD was evaluated in all groups.

Statistical analysis: Statistical analysis of this study was performed using IBM SPSS Statistics 22.0 package program. The normal distribution fit of the variables was examined with the One sample KolmogorovSmirnov test. Normally distributed variables are expressed as mean and standard error mean $(x \pm \mathrm{SD})$. Student's-T test was used for statistical analysis. Intergroup nanparametric data were analyzed using the Mann Witney U test. Pearson Correlation Coefficient was used to determine the direction and strength of the relationship between two quantitative variables. For statistical significance, a value of $\mathrm{p}<0.05$ was accepted.

\section{RESULTS}

In the statistical analysis made, Group 1 and Group 4, Group 2 and Group 3 show a homogeneous distribution in terms of age and gender distribution. It was found that the groups were not similar in the distribution between Group 1 and Group 2, Group 3 and Group $4(\mathrm{P}=0.001)$ (Table 1).

While there was a statistically significant difference in the vocal chorda distance of TD in the individuals in Group 1 and Group 2, no significant difference was found in the distance to the carina. While the average distance of TD of all individuals in both groups to the vocal chord is $5.75 \pm 1.57$, the average distance to the carina is $7.02 \pm 2.36$ (Table 2).

No statistically significant difference was found in the transverse and vertical magnitude of TD in individuals in Group 1 and Group 2. While the mean transverse size of the TDs of all individuals in both groups is 6.63 \pm 3.04 , the average vertical size is $19.10 \pm 7.24$ (Table 3 ).

It was observed that the vertebra levels of TDs in Group 1 and Group 2 were mostly at the T2 and T3 levels (Table 4).

The average of transverse and anteroposterior diameter lengths at T4 and T9 levels of all groups are shown in Table 5. In this evaluation, a statistically significant difference was found between Group 1 and Group 3 $(\mathrm{p}=0.016)(\mathrm{p}=0.017)$ only in the anteroposterior diameter at T4 and T9 levels.

\section{DISCUSSION}

In Group 2 (TD + COPD) the diverticulum is localized closer to the vocal cords, while in Group 1 (TD only) the diverticulum is formed further from the vocal cords. Therefore, COPD causes the diverticulum to be localized closer to the vocal cords by affecting the level of TD.

The fact that there is no difference in the transverse and vertical sizes of the diverticulum in the individuals in Group 1 and Group 2 shows that COPD has no effect on diverticulum dimensions and only affects the level on the trachea. In Group 1 and Group 2, we can interpret the result that diverticula are more common at T2 and T3 levels, as the structures supporting the trachea at these levels are weaker than other levels.

The significant difference in thorax vertical diameters at T4 and T9 levels between Group 1 (Only TD) and Group 3 (Only COPD) indicates that the thorax vertical length of the patients with COPD is greater than the patients with only TD.

The fact that there is no significant difference between the chest parameters in Group 3 (Only COPD) and Group 4 (Control) shows that COPD does not have a significant effect on the change of chest vertical distance. As a result of these evaluations, we can say that the vertical diameter of the chest is significantly 
less in patients with only TD compared to patients with COPD, and that TD reduces this diameter by adversely affecting the vertical distance of the chest in patients with COPD.

In the study of Kurt et al. (21), the mean age of patients with TD was 58, and according to Polat et al. (22), the mean age was $55 \pm 16,6$, and there was no significant correlation between TD and age. In another study, the average age of patients with TD was 59,8 (23).

In this study, the mean age in patients with TD was $47.31 \pm 14.88$, while it was $61.66 \pm 7.49$ in patients with TD + COPD. According to these studies, the incidence of TD is between the 5th and 6th decades, while the incidence of TD is between the 4th and the 6th decades in our study. The younger age of the TD patients in our study can be explained by the larger sample of patients screened.

TD has two subgroups, congenital and acquired. Congenital TD is more common in men than in women $(3,24)$. Accordingly Kurt et al. (21) TD is more common in men (64\%) than women (36\%). In the study of Cheng, Buterbough, and Huang, the reverse was more common in women than in men $(20,25,26)$. In this study, similar to Cheng, Buterbough, and Huang, the incidence of TD is higher in women than men, with a rate of $70,9 \%$.

Kokkonouzis et al. (11) reported that TD is usually located on the right posterolateral side of the trachea, and there are two forms of TD: congenital and acquired. According to the study performed by Goo et al. (27) on 65 patients with TD, $98 \%$ of the lesions were located in the right paratracheal region. The reason that the lesions are mostly seen on the right side is explained by the esophagus supporting the left side.

In the study of Kurt et al. (21), TD was reported to be generally located in the right posterolateral region with $97,1 \%$, and rarely on the left with $2,9 \%$. In the study of Gayerve et al. (24), TD was detected on the right side of the trachea. This situation is interpreted as the right side of the trachea is weaker due to the increased intratracheal pressure and the left side is more protected due to the support of the esophagus and arcus aorta. In another study, it was reported that TD can be seen along the right posterolateral wall near the thoracic inlet, however it can be seen at any level. It is emphasized that the reason for this is due to the origin of the cartilage deficiency in the obtained form, and the increased intraluminal pressure or the weakening of the structures after surgical procedures and damage to the mucous membrane. (28). Similarly, in this study, TD is located in the right posterolateral region in all patients. Diverticula were more common especially at T2 and T3 levels, due to the weaker structures supporting the trachea at this level compared to other levels.

Since the diverticulum usually acts as a reservoir for respiratory secretions, it is sometimes associated with chronic cough and can become infected (28). COPD and other inflammatory conditions can result in tracheomegaly or acquired TD. This is related to changes in the elastic properties of the respiratory tract (29). In a study by Kurt et al. (21) performed a retrospective chest CT scan and found bronchial diverticula and COPD in 84 of 412 TDs. The relationship between the localization of TD and bronchial COPD has been shown to be $2.38 \%$. According to the study of Goo et al. (30), coughing is the most common symptom in TD. It is reported that this symptom may be a sign of COPD in addition to associating it with only COPD and TD. Polat et al. (22) found a significant link between COPD and TD. There are two opposing views to these studies. The first of these, Buterbough et al. (20) showed that there was no relationship between emphysema and paratracheal air cysts. Second, in the study of Marina et al. (23), it was reported that TD is not associated with respiratory symptoms and COPD. In this study, when we compared the group with only TD and the group with COPD + TD, we found that COPD affected the presence of diverticula and there was a relationship between COPD and TD. When we evaluate it in terms of this result, this study is similar to the work of Kurt, Goo and Polat, but it also contradicts with the work of Buterbough and Marina.

The relationship between TD and tracheal lumen is shown in axial, coronal, and sagittal multiplan images (31). According to the study of Linn et al. (32), multidetector CT is the best method for imaging TD and it is a useful method for evaluating the localization, size, contour and wall thickness of TD. In order to evaluate $\mathrm{TD}$, the cross sectional thickness of the chest should be detailed more than about $1 \mathrm{~mm}$ (31). According to a study by Rahalkar et al. (16), the best method for the diagnosis of TD is CT scanning of the trachea and 
visualizing the communication of the cyst with the tracheal wall at various angles in the coronal plane.

In this study, tracheal diverticula are shown on CT with axial, coronal and sagittal multiplan images. Even if TD is very small, it can be diagnosed noninvasively with multi-slice CT. In addition, the boundaries of the diverticulum, its content and its connection with the tracheal lumen can be demonstrated with reconstruction without the need for fiberoptic bronchoscopy.

According to the study performed by Griscom et al. (33), many reasons may explain the increase in anteroposterior tracheal diameter, but COPD is the most dominant chronic inflammatory disease. Similarly, in this study, the average anteroposterior diameter values in patients with COPD were higher than in other groups. This difference is statistically higher than Group 1 with only TD. The fact that this difference is not statistically more than Group 4 (control) shows that COPD affects the anteroposterior diameter of the thorax, but does not make a significant difference. However, studies with more cases and investigating the effect of COPD on the anteroposterior diameter of the chest are also needed.

\section{CONCLUSION}

In this study, we concluded that by affecting the level of TD of COPD, diverticulum is formed in the upper levels of the trachea, and TDs are more common at T2 and T3 levels. Since there was no significant difference in the transverse and vertical sizes of TDs, we found that COPD had no effect on diverticulum dimensions, but COPD only affected the level of diverticulum. In addition, we concluded that TD reduces the vertical chest diameter in patients with COPD.

With all these results we found, we thought that TD had an effect on breast parameters in patients with COPD.

\section{REFERENCES}

1. Monroe MM, McLean M, Gross ND. Infectedtracheal diverticulum presenting as a cervical abscess Laryngoscope. 2013;123(7):1583-7. http://dx.doi.org/10,1002/lary.23978.

2. Smelley CL, Bowen R, Nathan CO. Intermittent lysymptomatic tracheal diverticulum: a case of a rare clinic alphenomenon. Ear Nose Throat J. 2011;90(9):10-2.

3 . Soto-Hurtado EJ, Pe-uela-Ruiz L, Rivera-Sanchez I, Torres-Jimenez J. Tracheal diverticulum: a review of theliterature. Lung. 2006;184(6):303-7. http://dx.doi. org/10.1007/s00408-006-0010-7.

4. Waite S, Sharma A, Machnicki S. Right paratracheal air cyst/ tracheal diverticulum. Appl Radiol. $2003 ; 32(8)$.

5. Shah M, Joshi JM. Tracheal Diverticulum. Indian J Chest Dis Allied Sci. 2012;54(1):39-40.

6 . Han S, Dikmen E, Aydin S, Yapakci O. Tracheal diverticulum: a rarecase of dysphagia. Eur J Cardiothorac Surg. 2008;34(4):916-7 http://dx.doi.org/10.1016/j. ejcts.2008.06.022.

7 . Haghi Z, Towhidi M, Fattahi H, Lari SM. Right paratracheal air cyst (tracheal diverticulum). Respir Care. 2009;54(10):1409-11.

8. Sharma BG. Tracheal diverticulum: a report of 4 cases. Ear Nose Throat J. 2009;88(1):E11.

9. Ortega HA, Vega NA, Comparini J, Carmo LV, Pereira, LP. Doencacisticatraqueobronquica. J Pneumol. 2001;27(6):349-54. http://dx.doi.org/10.1590/ S0102-35862001000600010.

10 . Charest M, Sirois C, Carter Y, Rousseau J. Infected tracheal diverticulum mimicking an aggressive mediastinallesion on FDG PET/CT: an interesting case withreview of the literature. $\mathrm{Br} \mathrm{J}$ Radiol. 2012;85(1009):e17-21. http:// dx.doi.org/10.1259/bjr/32814390.

11. Kokkonouzis I, Haramis D, Kornezos I, Moschouris H, Katsenos S, Bouchara S. Tracheal diverticulum in an asymptomaticmale: a casereport. Cases J. 2008;1(1):181. http://dx.doi.org/10.1186/1757-1626-1-181. 
12. Danielson GP, Jedlovszky V, Landigran GP. Tracheal diverticulum: a rare finding in a patient with worsening chroniccough. EarNoseThroat J. 2008;87(8):474-5.

13. Agusti EB, Vecina VM, Figuerola CR, Gaspa MM. Tracheal diverticulum: a case report. Acta Otorrinolaringol Esp. 2007;58(6):278-9. http://dx.doi.org/10.1016/ S2173-5735(07)70349-6.

14. Pinot D, Breen D, Peloni JM, Gaubert JY, Dutau H, Vervloet D. An incidental finding in a 34-year-old maleunder investigation for haemoptysis. Diagnosis: Theradiologicaland endoscopic images demonstrate a complex defect along the posterior tracheal Wall consistent with acquired tracheal diverticulum. Eur Respir J. 2009;33(5):1227-9. http://dx.doi.org/10.1183/09031936.00131908.

15. Elicora A, Liman ST, Topcu S, Akgul AG. Congenital tracheal diverticulum seen in adult age. Eur J Cardiothorac Surg. 2012;41(6):1399. http://dx.doi.org/10.1093/ejcts/ezr264.

16. Rahalkar MD, Lakhkar DL, Joshi SW, Gundawar S. Tracheal diverticula-report of 2 cases. Indian J RadiolImaging. 2004;14(2):197-8.

17. Soto PC, Congregado M, Loscertales J. Diverticulotraquealadquiridocomocausa de intubacionorotrachealcomplicada. Arch Bronconeumol. 2012;48(2):64-6. http:// dx.doi.org/10.1016/j.arbres.2011.09.006.

18. Djamouri F, Le PimpecBarthes F, Pheulpin G, Grignet JP, Carnot F, Riquet M Air-filled cysts of tracheal origin: nosological problem sand actual frequency [Article in French]. Rev Mal Respir. 2002;19(4):523-6.

19. Teh BM, Hall C, Kleid S. Infected tracheocoele (acquired tracheal diverticulum): case report and literature review. J Laryngol Otol. 2011;125(5):540-5. http://dx.doi. org/10.1017/S0022215110003026.

20. Buterbaugh JE, Erly WK. Paratrachealaircysts: a commonfinding on routine CT Examinations of the cervical spine and neck that maymimic pneumomediastinum in patients with traumatic injuries. AJNR Am J Neuroradiol. 2008;29(6):1218-21. http://dx.doi.org/10.3174/ajnr.A1058.

21. Kurt A, Sayit AT, Ipek A, et al. A multi detector computed tomography survey of tracheal diverticulum. Eurasian J Med 2013;45:145-8.

22. Polat AV, Elmali M, Aydin R, et al. Paratracheal air cysts: prevalence and correlation with lung diseases using multidetector CT. J Med Imaging Radiat Oncol 2014;58:144-8.

23. Marina P, Annarita D, Alessadre D, Alessio DG, Cesare M,Emanuele C,Francesco S, Sandro S. Tracheal Diverticula-A retrospective analyses of patients referred for thoracic ct.Observational study. Medicine. 2018 97:39.

24. Gayer G. Tracheal diverticula. Semin ultrsound CT. MRI 2016;37: 190-5.

25. Cheng HM, Chang PY, Chiang KH, et al. Prevalence and characteristics of paratracheal air cysts and their association with emphysema in a general population. Eur J Radiol 2012;81:2673-7.

26. Huang D, Ma Q, Wang S, et al. Transcervical resection of tracheal diverticulum. Head Neck 2017;39:18790 .

27. Goo JM, Im JG, Ahn JM, Moon WK, Chung JW, Park JH, et al. Right paratracheal air cysts in the thoracic inlet: clinical and radiologic significance. AJR Am J Roentgenol 1999;73(6):65-70.

28. Amaral CB, Silva S, Feijo S. Infected tracheal diverticulum: a rare association with alpha-1 antitrypsin deficiency. J Bras Pneumol . 2014;40(6):669-672. doi:10.1590/S1806-37132014000600011.

29. Flores-Franco RA, Silva-Alcaraz J. Acquired Tracheal Diverticulum as an Unexpected Cause of Endotracheal Tube Cuff Leak. J Crit Care Med (Targu Mures) . 2015;1(4):171-173. Published 2015 Nov 10. doi:10.1515/jccm-2015-0024.

30. MacKinnon D. Tracheal diverticula. J Pathol Bacteriol 1953;65(2):513-51. 
31. Tanrivermis Sayit A, Elmali M, Saglam D, Celenk C. The diseases of airway-tracheal diverticulum: a review of the literature. $J$ Thorac Dis . 2016;8(10):E1163-E1167. doi:10.21037/jtd.2016.10.92.

32 . Lin H, Cao Z, Ye Q. Tracheal diverticulum: a case report and literature review. Am J Otolaryngol 2014;35:542-5.

33. Griscom NT, Vawter GF, Stigol LC. Radiologicandpathologicabnormalities of thetrachea in olderpatientswithcysticfibrosis. Am J Roentgenol. 1987;148:691-3.

\section{Hosted file}

Tables.pdf available at https://authorea.com/users/393871/articles/507374-the-effect-oftracheal-diverticulum-and-chronic-ostructive-lung-disease-on-chest-anthropometry

\section{Hosted file}

Figures.pdf available at https://authorea.com/users/393871/articles/507374-the-effect-oftracheal-diverticulum-and-chronic-ostructive-lung-disease-on-chest-anthropometry 